\title{
Ranking of Research Output of Spanish Universities on the Basis of the Multidimensional Prestige of Influential Fields of Study
}

\author{
J. A. García, Rosa Rodriguez-Sánchez, \\ J. Fdez-Valdivia, Daniel Torres-Salinas, and \\ Francisco Herrera.
}

the date of receipt and acceptance should be inserted later

\begin{abstract}
A university may be considered as having dimension-specific prestige in a field of study (e.g., Computer Science) when a particular bibliometric research performance indicator exceeds a threshold value.

But a university has multidimensional prestige in a field of study only if it is influential with respect to a number of dimensions. The multidimensional prestige of influential fields at a given university takes into account that several prestige

J. A. García, Rosa Rodriguez-Sánchez, J. Fdez-Valdivia, and Francisco Herrera

Departamento de Ciencias de la Computación e I. A., CITIC-UGR, Universidad de Granada, 18071 Granada, Spain.

Address correspondence to J. A. García at jags@decsai.ugr.es

Daniel Torres-Salinas

EC3: Evaluación de la Ciencia y la Comunicación Científica, Centro de Investigación Médica Aplicada, Universidad de Navarra, 31008, Pamplona, Navarra, Spain
\end{abstract}


indicators should be used for a distinct analysis of the influence of a university in a particular field of study.

After having identified the multidimensionally influential fields of study at a university their prestige scores can be aggregated to produce a summary measure of the multidimensional prestige of influential fields at this university, which satisfies numerous properties. Here we use this summary measure of multidimensional prestige to assess the comparative performance of Spanish Universities during the period 2006-2010.

Keywords Publication-Based Ranking; Spanish Universities; Bibliometrics; Multidimensional Prestige; Influential Fields of Study

\section{Introduction}

The interest in the ranking of universities stems from the need to evaluate research output using to this aim some kind of objective metrics. For example, it may guide student choice of a university to pursue a graduate degree (Dridi et al., 2010).

The comparison of research output among universities has been raising an increasing amount of interest in the last few years (Liu and Cheng, 2005; Buela-Casal et al., 2007; Aguillo et al., 2010; Torres-Salinas et al., 2011), since the help it provides to allocate limited funds as fairly as possible. However funding agencies often make their decisions based on partial measures, resulting in unfair assessments of the research output of some of the studied universities (Billaut et al., 2010).

The number of papers produced in a year by each member of staff in an academic institution particularly in the EU and USA is regarded as an indication of their career success. Rankings based on publication in peer-reviewed journals are 
objective, and many faculty believe academic journals remain the fairest measure of the quality of our research (Dusansky and Vernon, 1998). Since publicationbased performance evaluations underlie the work of funding agencies, there are already mechanisms to ensure high levels of accuracy of these data (Dusansky and Vernon, 1998).

In (Torres-Salinas et al., 2011), it was presented a bidimensional quantitativequalitative index to compare the research output of a group of universities using different dimensions of analysis: (1) The quantitative dimension which shows the net production of a university in a given field during a period of time by using raw indicators that may be correlated with staff of the institution; and (2) the qualitative dimension which can be seen as a measurement for academic excellence, focusing on the ratio of high-quality production on each university in a particular field during the same period of time, and is mostly independent of the size of the institution. A combination of both dimensions provides a robust and objective way to compare research outputs.

In this paper we provide an overall ranking of research production in different fields of main Spanish universities based on a multivariate performance indicator space which integrates both quantitative and qualitative dimensions.

To this aim, we extend the one-dimensional measure developed by (Garcia et al., 2011a,b) to a multidimensional case following (Peichl and Pestel, 2010) who proposes a class of economic measures of richness in Germany. Thus our approach identifies those fields of study at a university that are considered to be multidimensionally influential. Furthermore, the multidimensional prestige of influential fields is to be sensitive to changes in the score distribution of each 
dimension, which allows us to investigate inequality among multidimensionally influential fields.

For example, let $U=\left\{s_{1}, s_{2}, \ldots, s_{n}\right\}$ be the set of fields of study at a given university of example. From (Torres-Salinas et al., 2011) we have that research output and impact of field $s_{i}$ at this university may be graded on the basis of the raw number of publications, citations, h-index, as well as relative measures of impact and visibility (e.g., JCR journal first quartile, average citations and ratio of highly cited papers).

Regarding the number of dimensions (prestige indicators) to be used in a multidimensional setting in order to measure research output and impact of influential fields at a particular university, we may consider several indicators with different degrees of correlation among them, but which should be used for a distinct analysis of structural changes at the score distribution of prestige in a given field of study, (Torres-Salinas et al., 2011):

- NDOC: Basic indicator for total amount of raw production, it may depend on the number of researchers in the institution focused on the field of study, and how active they are.

- NCIT, ACIT, TOPCIT: According to (Bornmann and Daniel, 2008), in bibliometrics the resonance, or impact, of a scientific work is measured via the number of citations. It can be assumed that the more important a work is for the further development of a field, the more frequently it is cited. That is, NCIT is a raw indicator of scientific relevance, and ACIT and TOPCIT indicate quality of the research output and ratio of very high-quality papers (Aksnes, 2003; Aksnes and Sivertsen, 2004), respectively. 
- H-index: Probably the better known index in current bibliometrics, it has proven to be a robust measure of impact, (Hirsch, 2005). By limiting its scope to the period of study, we avoid the seniority dependence the basic h-index usually presents.

- \%1Q: The impact factor is widely considered a reliable measure of journal quality (Bornmann and Daniel, 2008), so centering the analysis in the top quartile provides an indicator of top-quality papers. The ratio of citable papers that are top-quality serves as a relative size-independent indicator, $\% 1 \mathrm{Q}$.

In this paper, a field of study $s_{i}$ at a given university is considered as having dimension-specific prestige when its score based on a given ranking model (e.g., either NDOC or \%1Q ) exceeds a threshold value. Then, we can define which fields $s_{i}$ at a given university are considered to be prestigious in a multidimensional setting. Thus, a field of study at this university has multidimensional prestige only if it is an influential field with respect to a number of dimensions. Finally, after having identified the multidimensionally influential fields at a particular university, their prestige scores are aggregated to a summary measure of multidimensional prestige. The summary measure is not only sensitive to the number of dimensions but also takes into account changes in the ranking scores of influential fields of study at the university.

The setup of the paper is organized as follows: Section 2 defines the multidimensionally influential fields of study at a given university. The Section 3 introduces a summary measure of multidimensional prestige of influential fields, which satisfies numerous properties. Then in Section 4 we shall apply our approach to main universities in Spain in order to analyse the comparative multidimensional 
prestige of influential fields during the period 2006-2010. The data we employ is from (Torres-Salinas et al., 2011). Section 5 concludes.

\section{Multidimensionally influential fields of study at a given university}

The number of fields of study at a given university is denoted with $n$ as given above, and let $d \geq 2$ be the number of dimensions in the multivariate indicator space.

Let $\mathbf{X}$ be the matrix of dimension-specific scores $x_{i j}$ which denote the score of field of study $s_{i}$ at the particular university, with $1 \leq i \leq n$, in ranking model corresponding to dimension $j$, with $1 \leq j \leq d$ :

$$
\mathbf{X}=\left[x_{i j}\right]_{n \times d}
$$

For each dimension $j$, there is a threshold $z_{j}$ such that fields $s_{i}$ at this university with score $x_{i j}$ above threshold $z_{j}$ are to be considered dimension-specific influential fields of study.

Let $\mathbf{z}$ be the $1 \times d$ vector of dimension-specific thresholds. Using this vector it is possible identify whether field $s_{i}$ is influential with respect to dimension $j$ or not. Let $\theta_{i j}$ be a function defined as:

$$
\theta_{i j}=\left\{\begin{array}{l}
1 \text { if } x_{i j}>z_{j} \\
0 \text { otherwise }
\end{array}\right.
$$

Using function $\theta_{i j}$ it is possible to construct a matrix $\Theta^{0-1}$ which provides information about whether a field of study $s_{i}$ at the given university is influential regarding dimension $j$ or not: 


$$
\Theta^{0-1}=\left[\theta_{i j}\right]_{n \times d}
$$

where each row vector $\theta_{i}$ of $\Theta^{0-1}$ gives us a vector of prestige counts which can be denoted as $\mathbf{c}=\left(c_{1}, \cdots, c_{n}\right)^{\prime}$ whose elements $c_{i}=\sum_{j=1}^{d} \theta_{i j}$ are equal to the number of dimensions in which field of study $s_{i}$ is found to be prestigious.

We can now define which fields of study at a university are considered to be influential in a multidimensional sense: A field of study $s_{i}$ at the given university is a multidimensionally influential field if it is prestigious for a number of dimensions which is greater than or equal to a certain integer $k$, with $1 \leq k \leq d$.

That is, a field $s_{i}$ is multidimensionally influential if $c_{i} \geq k$, with $c_{i}$ being the number of dimensions in which field of study $s_{i}$ at the university was found to be influential.

For a given integer $k$, we can define a function $\phi_{i}(\mathbf{z} ; k)$ which equals to one if field $s_{i}$ is multidimensionally influential, and is zero otherwise:

$$
\phi_{i}(\mathbf{z} ; k)=\left\{\begin{array}{l}
1 \text { if } c_{i} \geq k \\
0 \text { otherwise }
\end{array}\right.
$$

with $\mathbf{z}$ being the $1 \times d$ vector of dimension-specific thresholds.

Therefore the subset of fields of study at the university which are multidimensionally influential is given by:

$$
\Phi(\mathbf{z} ; k)=\left\{s_{i}, 1 \leq i \leq n \mid \phi_{i}(\mathbf{z} ; k)=1\right\}
$$

For a given integer $k$, let $w(k)$ be the number of multidimensionally influential fields at this university. From equation (5) it follows that $w(k)$ is given by the cardinal of the subset $\Phi(\mathbf{z} ; k)$ : 


$$
w(k)=|\Phi(\mathbf{z} ; k)|
$$

where $|\cdot|$ is the cardinality (size) of a set.

In case of $k=1$, field of study $s_{i}$ is multidimensionally influential when it is considered prestigious in only one single dimension (e.g., \%1Q). But prestige in one single dimension may be something dangerous (Garcia et al., 2011c).

Second, in case of $k=d$, it is only considered as multidimensionally influential if it is prestigious for all dimensions under consideration. But this is a demanding requirement, especially if the number of dimensions $d$ of the multivariate indicator space is large, which often identifies a very narrow slice of fields at the university under consideration.

In case of $1<k<d$ we have an intermediate approach as proposed in (Alkire and Foster, 2008).

\section{A summary measure of multidimensional prestige}

Recall that the vector of prestige counts denoted as $\mathbf{c}$ was defined such that $\mathbf{c}=$

$\left(c_{1}, \cdots, c_{n}\right)^{\prime}$, where $c_{i}=\sum_{j=1}^{d} \theta_{i j}$ is the number of dimensions in which field of study $s_{i}$ is found to be prestigious, with $\theta_{i j}$ being equal to one if field $s_{i}$ is prestigious with respect to dimension $j$ and zero otherwise as given in equation (2). Since a summary measure of the multidimensional prestige of influential fields at the university must take into account information on multidimensionally influential fields of study only, we must replace the elements of $\mathbf{c}$ as follows: 


$$
c_{i}^{k}=\left\{\begin{array}{l}
c_{i} \text { if } c_{i} \geq k \\
0 \text { otherwise }
\end{array}\right.
$$

From equation (7), we have that $\mathbf{c}^{\mathbf{k}}=\left(c_{1}^{k}, \cdots, c_{i}^{k}, \cdots, c_{n}^{k}\right)^{\prime}$ contains zeros for fields $s_{i}$ not considered to be multidimensionally prestigious, that is, when a field of study $s_{i}$ is not multidimensionally influential, $c_{i}<k$, its entry in $\mathbf{c}^{\mathbf{k}}$ is zero.

Now we propose a number of constraints which an axiomatic measure of the multidimensional prestige of influential fields at a given university must satisfy. But first, following the approach given in (Garcia et al., 2011a), we define a summary measure $M W$ of the multidimensional prestige of influential fields at the university as the normalized weighted sum of the field contribution to the overall prestige as follows:

Definition 1 Given a configuration $\mathbf{X}=\left[x_{i j}\right]_{n \times d}$ of dimension-specific scores of size $n \times d$, and a $1 \times d$ vector $\mathbf{z}=\left(\mathbf{z}_{1}, \ldots, \mathbf{z}_{\mathbf{j}}, \ldots, \mathbf{z}_{\mathbf{d}}\right)$ of dimension-specific thresholds, a summary measure of the overall prestige $M W$ of multidimensionally influential fields at a given university is defined by a normalized weighted sum of field contributions to the overall prestige using weighting function $f$, as follows:

$$
M W=\frac{1}{n \times d} \sum_{i=1}^{n} \sum_{j=1}^{d} f\left(\frac{x_{i j}}{z_{j}}\right),
$$

where the mathematical form of $f$ depends on a set of axioms to be proposed.

Appendix A presents a set of axioms in order to define the exact form of a summary measure as that given in Definition 1 which shall have some desirable properties. To this aim we reformulate to the study of the multidimensional 
prestige of influential fields a number of constraints which were first used in an axiomatic approach to economic poverty measurement (Sen, 1976; Takayama, 1979; Peichl et al., 2008).

Next, following (Garcia et al., 2011c), a theorem states that five axioms given in Appendix A determine an axiomatic measure of multidimensional prestige of influential fields for a given domain-specific score configuration.

Theorem 1 Let $k$ be such that field of study $s_{i}$ at a given university is multidimensionally influential if $c_{i} \geq k$, with $c_{i}$ being the number of dimensions in which field $s_{i}$ was found to be influential. Then, a summary measure of the multidimensional prestige of influential fields, given by a normalized weighted sum of domain-specific scores in the configuration $\mathbf{X}$ of size $n \times d$, using a weighting function $f$ as follows:

$$
\frac{1}{n \times d} \sum_{i=1}^{n} \sum_{j=1}^{d} f\left(\frac{x_{i j}}{z_{j}}\right)
$$

and such that satisfies Axioms 1 through 5 in Appendix A, it can be defined as:

$$
M W(k)=\frac{1}{n \times d} \sum_{i=1}^{n} \sum_{j=1}^{d}\left(1-\left(\frac{z_{j}}{x_{i j}}\right)^{\beta}\right)_{+} \cdot \phi_{i}(\mathbf{z} ; k)
$$

with $\beta>0$ being a sensitivity parameter for the intensity of field prestige (for smaller values of $\beta$ more weight is put on more intense prestige); $(y)_{+}=$ $\max (y, 0)$; and where function $\phi_{i}(\mathbf{z} ; k)$ equals to one if field $s_{i}$ is multidimensionally influential, and is zero otherwise.

Proof See Appendix B. 


\begin{tabular}{|c|c|c|c|}
\hline \multicolumn{4}{|c|}{ Spanish Universities } \\
\hline Alcala & Alicante & Almeria & Aut. Barcelona \\
\hline Aut. Madrid & Barcelona & Burgos & Cadiz \\
\hline Cantabria & Card. Herrera CEU & Carlos III & Cartagena \\
\hline Castilla la Mancha & Complutense Madrid & Córdoba & Coruña \\
\hline Deusto & Europea de Madrid & Extremadura & Girona \\
\hline Granada & Huelva & Baleares & Jaen \\
\hline Jaume I & La Laguna & La Rioja & Las Palmas \\
\hline León & Lleida & Mondragón & Oviedo \\
\hline Pais Vasco & Polit. Cataluña & Polit. Madrid & Polit. Valencia \\
\hline Pontificia de Comillas & Málaga & Miguel Hernndez & Murcia \\
\hline Navarra & P. Navarra & Pablo Olavide & Pompeu Fabra \\
\hline Salamanca & Ramón Llull & Rey Juan Carlos & Rovira i Virgil \\
\hline San Pablo-Ceu & Santiago Compostela & Sevilla & UNED \\
\hline Valencia & Valladolid & Vigo & Zaragoza \\
\hline
\end{tabular}

Table 1 Set of 56 Spanish universities which was chosen to perform the comparison of research output during the period 2006-2010

\section{Ranking of Spanish universities}

Here we show the ranking of research output of Spanish universities during the period 2006-2010. To this aim we compute the multidimensional prestige of influential fields of study at each institution using a multivariate indicator space.

4.1 Dimensions of the multivariate indicator space

Six variables are candidates to be used in this analysis, (Torres-Salinas et al., 2011): 1) Raw number of citable papers published in scientific journals (NDOC); 2) Number of citations received by all impact citable papers (NCIT); 3) H-Index 
$(\mathrm{H})$; 4) Ratio of papers published in journals in the top JCR quartile $\frac{100 \times N 1 Q}{N D O C}$

(\%1Q ); 5) Average number of citations received by all citable papers (ACIT); and

6) Ratio of papers that belong to the top $10 \%$ most cited (TOPCIT).

Once the set of Spanish universities was chosen (see Table 1), along with a period of time (2006-2010), the research output of each university indexed in the Science Citation Index of the ISI-Web of Knowledge (http://isiknowledge.co) was retrieved using the field "Address" as a filter and taking into account all the different names each university receives, (Torres-Salinas et al., 2011). Next, the production of each one of the universities within different fields of study is extracted. The number $n$ of fields at each university may be lesser than or equal to $19(n \leq 19)$. Table 2 illustrates the 19 fields of study which were used in this analysis.

A scientific work is considered to be part of a field if it was published in a journal indexed in one of the JCR journal categories in this particular field of study. In order to calculate the indicators related to journal Impact Factor, the editions of the JCRs for the period of time of interest should be used. The data were downloaded in September 2011.

Table 4 in (Torres-Salinas et al., 2011) shows correlation analysis among six bibliometric indicators (i.e., NDOC, NCIT, H, \%1Q, ACIT, and TOPCIT) using data from the top $75 \%$ Spanish universities in 2000-2009. In general, it turns out that the quantitative indicators (i.e., NDOT, NCIT, and H ) are positively correlated as expected, and also, but to a lesser degree, there are correlations within the qualitative ones (i.e., \%1Q, ACIT, and TOPCIT). The correlations between a quantitative indicator and a qualitative one are in general very low. Thus, following (Torres-Salinas et al., 2011) we consider that this correlation is low 


\begin{tabular}{|c|c|}
\hline \multicolumn{2}{|r|}{ Fields of Study } \\
\hline$i$ & Name \\
\hline 1 & Agriculture \\
\hline 2 & Biology \\
\hline 3 & Biochemistry, Cell and Molecular Biology \\
\hline 4 & Food Science and Technology \\
\hline 5 & Materials Science \\
\hline 6 & Ecology and Environmental Sciences \\
\hline 7 & Pharmacoloy and Toxicology \\
\hline 8 & Genetics and Evolutionary Biology \\
\hline 9 & Geosciences \\
\hline 10 & Computer Science \\
\hline 11 & Chemical Engineering \\
\hline 12 & Medicine \\
\hline 13 & Microbiology and Virology \\
\hline 14 & Multidisciplinary Sciences \\
\hline 15 & Neurosciences \\
\hline 16 & Psychology \\
\hline 17 & Chemistry \\
\hline 18 & Public Health \\
\hline 19 & Veterinary \\
\hline
\end{tabular}

Table 2 Fields of study which were considered in the analysis of research output of each university

enough to conclude that quantitative and qualitative indicators describe different aspects of information without loss of interpretability, as happens when using variables obtained from a Principal Component Analysis.

From these results, we define the six dimensions of the multivariate indicator space as follows: $(\mathrm{j}=1)$ NDOC ; $(\mathrm{j}=2)$ NCIT; $(\mathrm{j}=3) \mathrm{H}$-index; $(\mathrm{j}=4) \% 1 \mathrm{Q} ;(\mathrm{j}=5)$ 
ACIT; and $(j=6)$ TOPCIT. Then we have that the number of dimensions in the multivariate indicator space is $d=6$.

For each dimension of the multivariate indicator space we must define a threshold such that fields of study at a given university with ranking score above this threshold are to be considered dimension-specific influential fields. More precisely, given a dimension-specific threshold $z_{j}$ as well as scores $x_{i j}$ which denote the ranking score of field $s_{i}$ corresponding to dimension $j$, we have that fields of study $s_{i}$ with ranking score $x_{i j}$ above threshold $z_{j}$ are dimension-specific influential fields.

For example, thresholds $z_{j}$, with $j=1, \cdots, 6$, can be defined such that the top $30 \%$ of the score distribution given by the corresponding ranking model (over all Spanish universities under consideration) are dimension-specific influential.

Recall that a field of study $s_{i}$ at a given university is defined multidimensionally influential if it is prestigious with respect to a number of dimensions which is greater than or equal to a certain integer $k$, with $1 \leq k \leq d$. But in case of $k=1$, $s_{i}$ is multidimensionally prestigious when it is considered prestigious in only one dimension which can be something dangerous, (Garcia et al., 2011c). On the other hand, in case of $k=d$, it is only considered as multidimensionally influential if it is prestigious in all dimensions under consideration which is a demanding requirement and often identifies a very narrow slice of fields.

If we choose larger values for thresholds $z_{j}$ and integer $k$ (e.g., $k=4$ and thresholds $z_{j}$ are such that the top $10 \%$ of the score distribution given by the corresponding ranking model are prestigious), we have that the ranking of Spanish universities will be based on more elitist principles. By the contrary if the values of thresholds $z_{j}$ and $k$ decrease (e.g., $k=2$ and the top $40 \%$ of the score distribution), it follows a more comprehensive analysis. 


\begin{tabular}{|c||c|c|c|c|c|c|c|c|c|c|c|c|c||c||c|}
\hline \multicolumn{10}{|c|}{ University of Granada } \\
\hline$i$ & $N D O C$ & $N C I T$ & $H$ & $\% 1 Q$ & $A C I T$ & \multicolumn{1}{|c|}{ TOPCIT } & \multicolumn{3}{|c|}{$\Theta^{0-1}$} & $c_{i}$ & $\phi_{i}(z ; k)$ \\
\hline 1 & 144 & 729 & 13 & 0.7290 & 5.0630 & 0.1250 & 0 & 0 & 0 & 1 & 0 & 1 & 2 & 1 \\
\hline 2 & 324 & 1310 & 15 & 0.4040 & 4.0430 & 0.1110 & 1 & 1 & 1 & 0 & 0 & 0 & 3 & 1 \\
\hline 3 & 353 & 2399 & 23 & 0.3140 & 6.7960 & 0.0930 & 1 & 1 & 1 & 0 & 1 & 0 & 4 & 1 \\
\hline 4 & 161 & 776 & 15 & 0.6960 & 4.8200 & 0.1060 & 1 & 1 & 1 & 1 & 0 & 0 & 4 & 1 \\
\hline 5 & 156 & 934 & 16 & 0.6600 & 5.9870 & 0.1790 & 1 & 1 & 1 & 1 & 0 & 1 & 5 & 1 \\
\hline 6 & 375 & 2096 & 18 & 0.4720 & 5.5890 & 0.1200 & 1 & 1 & 1 & 0 & 0 & 1 & 4 & 1 \\
\hline 7 & 190 & 1373 & 17 & 0.3160 & 7.2260 & 0.1370 & 1 & 1 & 1 & 0 & 1 & 1 & 5 & 1 \\
\hline 8 & 100 & 487 & 11 & 0.3500 & 4.8700 & 0.0300 & 0 & 0 & 0 & 0 & 0 & 0 & 0 & 0 \\
\hline 9 & 705 & 2962 & 19 & 0.5480 & 4.2010 & 0.0950 & 1 & 1 & 1 & 0 & 0 & 0 & 3 & 1 \\
\hline 10 & 484 & 2165 & 20 & 0.3310 & 4.4730 & 0.1710 & 1 & 1 & 1 & 0 & 0 & 1 & 4 & 1 \\
\hline 11 & 102 & 374 & 10 & 0.6080 & 3.6670 & 0.0690 & 0 & 0 & 0 & 1 & 0 & 0 & 1 & 0 \\
\hline 12 & 815 & 5298 & 30 & 0.4090 & 6.5010 & 0.0960 & 1 & 1 & 1 & 0 & 1 & 0 & 4 & 1 \\
\hline 13 & 207 & 1297 & 17 & 0.3140 & 6.2660 & 0.0920 & 1 & 1 & 1 & 0 & 1 & 0 & 4 & 1 \\
\hline 14 & 36 & 709 & 13 & 0.8330 & 19.6940 & 0.0280 & 0 & 0 & 0 & 1 & 1 & 0 & 2 & 1 \\
\hline 15 & 232 & 1308 & 17 & 0.3230 & 5.6380 & 0.0780 & 1 & 1 & 1 & 0 & 0 & 0 & 3 & 1 \\
\hline 16 & 362 & 1176 & 15 & 0.2210 & 3.2490 & 0.1220 & 1 & 1 & 1 & 0 & 0 & 1 & 4 & 1 \\
\hline 17 & 857 & 5019 & 25 & 0.5640 & 5.8560 & 0.0690 & 1 & 1 & 1 & 0 & 0 & 0 & 3 & 1 \\
\hline 18 & 94 & 378 & 9 & 0.3620 & 4.0210 & 0.0960 & 0 & 0 & 0 & 0 & 0 & 0 & 0 & 0 \\
\hline 19 & 15 & 47 & 3 & 0.8000 & 3.1330 & 0.1330 & 0 & 0 & 0 & 1 & 0 & 1 & 2 & 1 \\
\hline
\end{tabular}

Table 3 (First column) lists fields of study ordered as given in Table 2; (second column)

NDOC, NCIT, H, \%1Q, ACIT, and TOPCIT; (third column) $\theta_{i j}$ equals to one if field $s_{i}$ is prestigious with respect to dimension $j$ and zero otherwise; (fourth column) lists prestige counts $c_{i}=\sum_{j=1}^{d} \theta_{i j}$ that represents the number of dimensions in which field $s_{i}$ is found to be influential; (fifth column) shows $\phi_{i}(\mathbf{z} ; k)$ values which equal to one if field $s_{i}$ is multidimensionally influential and is zero otherwise.

An intermediate approach corresponds to the situation in which, for example, $k=2$ and thresholds $z_{j}$ are such that the top $30 \%$ of the score distribution given by the corresponding ranking model are dimension-specific influential.

\subsection{Multidimensional prestige of influential fields at the University of Granada}

In this section, we illustrate the measurement of the multidimensional prestige of influential fields at the University of Granada. 
Table 3 (second column) provides information on the one-dimensional score distributions of the six dimensions under consideration: $(j=1)$ NDOC ; $(j=2)$ NCIT; $(\mathrm{j}=3) \mathrm{H}$-index; $(\mathrm{j}=4) \% 1 \mathrm{Q} ;(\mathrm{j}=5)$ ACIT; and $(\mathrm{j}=6)$ TOPCIT. Table 3 (first column) lists the 19 fields of study ordered as given in Table 2.

The multidimensional prestige $M W(k)$ was computed for $k=2$ and thresholds $z_{j}$, with $j=1, \cdots, 6$, such that only the top $30 \%$ of the score distribution given by the corresponding ranking model in each dimension are dimension-specific influential. In this case we have that $z_{1}=148 ; z_{2}=748 ; z_{3}=13 ; z_{4}=0.57 ; z_{5}=6.13$; and $z_{6}=0.11$. The value of $\beta$ in equation (10) is $\beta=3$ following the results presented in (Garcia et al., 2011a,b).

For this same university, Table 3 (fourth column) lists prestige counts $c_{i}=$ $\sum_{j=1}^{d} \theta_{i j}$ which represent the number of dimensions in which field of study $s_{i}$ is found to be influential, with $\theta_{i j}$ being equal to one if field $s_{i}$ is prestigious with respect to dimension $j$ and zero otherwise as given in equation (2) (third column in Table 3).

Table 3 (fifth column) shows $\phi_{i}(\mathbf{z} ; k)$ values which equal to one if field of study $s_{i}$ (at the University of Granada) is multidimensionally influential and is zero otherwise, as given in equation (4). Recall that we select $k=2$ for this example of application.

Table 4 lists the $\theta_{i j}^{\beta}(k)$ values which are defined as:

$$
\theta_{i j}^{\beta}(k)=\left(1-\left(\frac{z_{j}}{x_{i j}}\right)^{\beta}\right)_{+} \cdot \phi_{i}(\mathbf{z} ; k)
$$

Fig. 1 illustrates the computation of the elements $\theta_{i j}^{\beta}(k)$ for a field of study $s_{i}$ at a given university. 


\begin{tabular}{|c|c|c|c|c|c|c|}
\hline \multicolumn{7}{|c|}{ University of Granada } \\
\hline & \multicolumn{6}{|c|}{$\theta^{\beta}{ }_{i j}(k)$} \\
\hline$i$ & $j=1$ & $j=2$ & $j=3$ & $j=4$ & $j=5$ & $j=6$ \\
\hline 1 & 0.0000 & 0.0000 & 0.0000 & 0.5220 & 0.0000 & 0.1800 \\
\hline 2 & 0.9047 & 0.8138 & 0.3490 & 0.0000 & 0.0000 & 0.0000 \\
\hline 3 & 0.9263 & 0.9697 & 0.8194 & 0.0000 & 0.2650 & 0.0000 \\
\hline 4 & 0.2232 & 0.1044 & 0.3490 & 0.4507 & 0.0000 & 0.0000 \\
\hline 5 & 0.1461 & 0.4864 & 0.4636 & 0.3558 & 0.0000 & 0.7207 \\
\hline 6 & 0.9385 & 0.9546 & 0.6233 & 0.0000 & 0.0000 & 0.0731 \\
\hline 7 & 0.5274 & 0.8383 & 0.5528 & 0.0000 & 0.3886 & 0.3771 \\
\hline 8 & 0.0000 & 0.0000 & 0.0000 & 0.0000 & 0.0000 & 0.0000 \\
\hline 9 & 0.9907 & 0.9839 & 0.6797 & 0.0000 & 0.0000 & 0.0000 \\
\hline 10 & 0.9714 & 0.9588 & 0.7254 & 0.0000 & 0.0000 & 0.6797 \\
\hline 11 & 0.0000 & 0.0000 & 0.0000 & 0.0000 & 0.0000 & 0.0000 \\
\hline 12 & 0.9940 & 0.9972 & 0.9186 & 0.0000 & 0.1604 & 0.0000 \\
\hline 13 & 0.6345 & 0.8082 & 0.5528 & 0.0000 & 0.0623 & 0.0000 \\
\hline 14 & 0.0000 & 0.0000 & 0.0000 & 0.6796 & 0.9698 & 0.0000 \\
\hline 15 & 0.7404 & 0.8130 & 0.5528 & 0.0000 & 0.0000 & 0.0000 \\
\hline 16 & 0.9317 & 0.7427 & 0.3490 & 0.0000 & 0.0000 & 0.1180 \\
\hline 17 & 0.9948 & 0.9967 & 0.8594 & 0.0000 & 0.0000 & 0.0000 \\
\hline 18 & 0.0000 & 0.0000 & 0.0000 & 0.0000 & 0.0000 & 0.0000 \\
\hline 19 & 0.0000 & 0.0000 & 0.0000 & 0.6383 & 0.0000 & 0.3192 \\
\hline$\frac{1}{n} \sum_{i=1}^{n} \theta_{i j}^{\beta}(k)$ & 0.5223 & 0.5509 & 0.4103 & 0.1393 & 0.0972 & 0.1299 \\
\hline
\end{tabular}

Table 4 (First column) lists fields of study at the University of Granada ordered as given in

Table 2 ; (second column) lists $\theta_{i j}^{\beta}(k)$ elements given in equation (11).

Since, from equation (10), the summary measure $M W(k)$ of multidimensional prestige of influential fields at a given university is equal to the sum of elements $\theta_{i j}^{\beta}(k)$ divided by the value $n \times d$, it follows that $M W(k)=0.3083$ for the University 


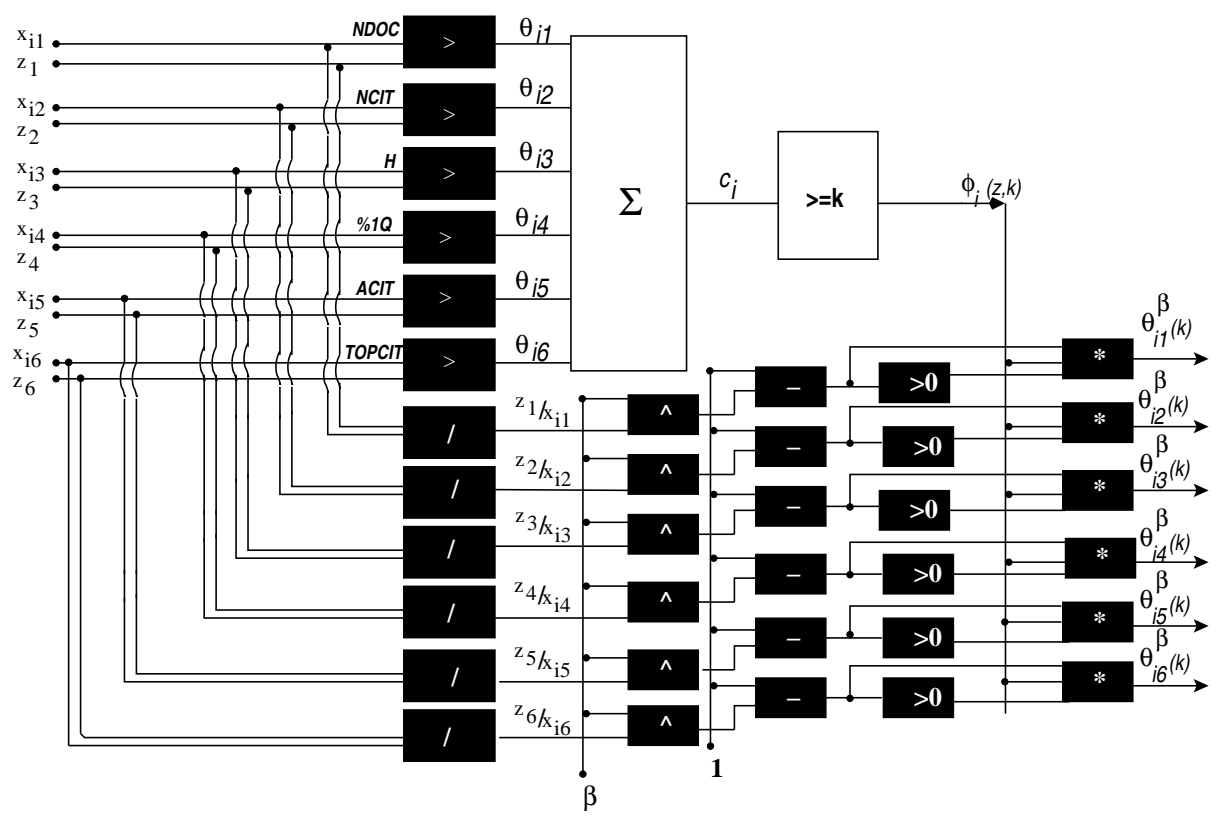

Fig. 1 Computation of $\theta_{i j}^{\beta}(k)$ values for a field of study $s_{i}$ at a given university.

of Granada. Again, Table 4 (first column) lists fields of study ordered as given in Table 2 .

In addition to looking at the overall value of multidimensional prestige of influential fields at the University of Granada, we can provide information on how different dimensions of the multivariate indicator space contribute to the measure $M W(k)$ of multidimensional prestige. To this aim, we rewrite equation (10) as follows:

$$
M W(k)=\frac{1}{d} \sum_{j=1}^{d} \frac{\sum_{i=1}^{n} \theta_{i j}^{\beta}(k)}{n}=\frac{1}{d} \sum_{j=1}^{d} \Pi_{j}^{\beta}(k)
$$

where $\Pi_{j}^{\beta}(k)=\frac{1}{n} \sum_{i=1}^{n} \theta_{i j}^{\beta}(k)$ represents the contribution of each dimension $j$ (multiplied by the number $d$ of dimensions) to the measurement of multidimensional prestige of influential fields. 
To the University of Granada, from Table 4 (bottom) we have that the contribution $\Pi_{j}^{\beta}(k)$ of the NCIT dimension $(j=2)$ is about $29.7 \%$ of the multidimensional prestige, and taken together, the NDOC, NCIT, and $\mathrm{H}$ dimensions make up about $80.19 \%$ of the multidimensional prestige of influential fields of study at this university. Hence, the NDOC, NCIT and H dimensions play a dominant role to the measurement of the multidimensional prestige $M W(k)$ for the University of Granada.

\subsection{Results}

In this section, we use the summary measure of multidimensional prestige $M W(k)$ to assess the comparative performance of selected Spanish universities during the period 2006-2010. Fifty-six main universities in Spain are considered in this experiment.

Tables 5 and 6 show the ranking of the 56 Spanish universities according to the multidimensional prestige $M W(k)$ of influential fields of study, for different selections of $k$ and thresholds $z_{j}$ with $j=1, \cdots, 6$. For our analysis the university with the best value of the multidimensional prestige of influential fields is assigned the rank \#1, the second best \#2, and so.

In order to produce the results given in Tables 5 and 6 , thresholds $z_{j}$ with $j=1, \cdots, 6$ were defined such that the top $20 \%$ (or alternatively $30 \%$, and $40 \%$ ) of the score distribution given by the corresponding journal ranking model (over all selected Spanish universities) are dimension-specific influential. For example, in case of the top $30 \%$ we have that $z_{1}=148 ; z_{2}=748 ; z_{3}=13 ; z_{4}=0.57 ; z_{5}=6.13$; and $z_{6}=0.11$ 


\begin{tabular}{|c|c|c|c|c|c|c|c|c|c|c|}
\hline \multicolumn{11}{|c|}{ Ranking of Spanish Universities } \\
\hline \multirow{2}{*}{ University } & \multicolumn{3}{|c|}{$k=2$} & \multicolumn{3}{|c|}{$k=3$} & \multicolumn{3}{|c|}{$k=4$} & \multirow{2}{*}{ Median } \\
\hline & $20 \%$ & $30 \%$ & $40 \%$ & $20 \%$ & $30 \%$ & $40 \%$ & $20 \%$ & $30 \%$ & $40 \%$ & \\
\hline BARCELONA & 1 & 1 & 1 & 1 & 1 & 1 & 1 & 1 & 1 & 1 \\
\hline AUTÓNOMA DE BARCELONA & 2 & 2 & 2 & 2 & 2 & 2 & 2 & 2 & 2 & 2 \\
\hline COMPLUTENSE DE MADRID & 3 & 3 & 3 & 5 & 3 & 3 & 6 & 4 & 5 & 3 \\
\hline VALENCIA & 4 & 4 & 4 & 3 & 4 & 4 & 3 & 3 & 4 & 4 \\
\hline AUTÓNOMA DE MADRID & 5 & 5 & 5 & 4 & 5 & 5 & 5 & 5 & 3 & 5 \\
\hline POMPEU FABRA & 6 & 7 & 8 & 6 & 6 & 8 & 4 & 6 & 8 & 6 \\
\hline GRANADA & 7 & 6 & 7 & 7 & 7 & 7 & 25 & 10 & 6 & 7 \\
\hline SANTIAGO DE COMPOSTELA & 8 & 8 & 6 & 8 & 8 & 6 & 15 & 9 & 7 & 8 \\
\hline SEVILLA & 10 & 10 & 9 & 9 & 9 & 9 & 21 & 7 & 9 & 9 \\
\hline ZARAGOZA & 11 & 9 & 10 & 10 & 10 & 10 & 12 & 8 & 10 & 10 \\
\hline POLITÉCNICA DE VALENCIA & 9 & 11 & 11 & 11 & 11 & 12 & 8 & 11 & 12 & 11 \\
\hline ROVIRA I VIRGILI & 16 & 13 & 13 & 15 & 12 & 13 & 20 & 15 & 13 & 13 \\
\hline CASTILLA-LA MANCHA & 19 & 14 & 12 & 19 & 15 & 11 & 32 & 14 & 11 & 14 \\
\hline CÓRDOBA & 12 & 18 & 16 & 12 & 16 & 16 & 7 & 12 & 14 & 14 \\
\hline VIGO & 13 & 12 & 14 & 16 & 13 & 15 & 10 & 16 & 15 & 14 \\
\hline PAÍS VASCO & 17 & 16 & 15 & 13 & 14 & 14 & 27 & 21 & 19 & 16 \\
\hline MURCIA & 20 & 22 & 22 & 17 & 19 & 17 & 16 & 13 & 16 & 17 \\
\hline REY JUAN CARLOS & 14 & 15 & 18 & 14 & 17 & 18 & 14 & 17 & 21 & 17 \\
\hline ISLAS BALEARES & 23 & 19 & 17 & 24 & 18 & 19 & 17 & 20 & 20 & 19 \\
\hline MIGUEL HERNÁNDEZ & 22 & 21 & 19 & 18 & 21 & 20 & 19 & 18 & 17 & 19 \\
\hline OVIEDO & 18 & 23 & 21 & 20 & 23 & 22 & 9 & 22 & 23 & 22 \\
\hline JAUME I DE CASTELLÓN & 15 & 17 & 23 & 27 & 22 & 24 & 18 & 23 & 31 & 23 \\
\hline NAVARRA & 28 & 24 & 25 & 23 & 20 & 25 & 11 & 19 & 22 & 23 \\
\hline LLEIDA & 25 & 20 & 20 & 36 & 26 & 21 & 46 & 24 & 25 & 25 \\
\hline SALAMANCA & 24 & 26 & 26 & 21 & 25 & 26 & 13 & 25 & 18 & 25 \\
\hline POLITÉCNICA DE CATALUÑA & 21 & 25 & 28 & 22 & 24 & 27 & 49 & 40 & 30 & 27 \\
\hline ALICANTE & 29 & 29 & 30 & 28 & 28 & 32 & 22 & 26 & 27 & 28 \\
\hline
\end{tabular}

Table 5 Ranking of Spanish universities during the period 2006-2010 according to the multidimensional prestige $M W(k)$ of influential fields, for different selections of $k$ and thresholds $z_{j}$.

Regarding the value of $k$, here we follow an intermediate approach, and thus, a field $s_{i}$ at a given university is defined multidimensionally influential if it is prestigious with respect to a number of dimensions which is greater than or equal to a certain integer $k$ with $1<k<6$. Thus the multidimensional prestige $M W(k)$ was computed for different values of $k$, with $k=2,3$, and 4 . 


\begin{tabular}{|c|c|c|c|c|c|c|c|c|c|c|}
\hline \multicolumn{11}{|c|}{ Ranking of Spanish Universities } \\
\hline \multirow{2}{*}{ University } & \multicolumn{3}{|c|}{$k=2$} & \multicolumn{3}{|c|}{$k=3$} & \multicolumn{3}{|c|}{$k=4$} & \multirow{2}{*}{ Median } \\
\hline & $20 \%$ & $30 \%$ & $40 \%$ & $20 \%$ & $30 \%$ & $40 \%$ & $20 \%$ & $30 \%$ & $40 \%$ & \\
\hline LA LAGUNA & 31 & 28 & 24 & 26 & 29 & 23 & 43 & 31 & 24 & 28 \\
\hline ALCALÁ DE HENARES & 26 & 27 & 29 & 29 & 27 & 29 & 31 & 27 & 32 & 29 \\
\hline MÁLAGA & 33 & 34 & 27 & 33 & 36 & 28 & 30 & 29 & 26 & 30 \\
\hline EXTREMADURA & 30 & 32 & 32 & 30 & 31 & 31 & 40 & 28 & 28 & 31 \\
\hline PABLO DE OLAVIDE & 32 & 31 & 35 & 38 & 32 & 34 & 24 & 30 & 34 & 32 \\
\hline GIRONA & 39 & 36 & 33 & 39 & 33 & 33 & 26 & 34 & 33 & 33 \\
\hline CANTABRIA & 27 & 30 & 34 & 25 & 34 & 37 & 33 & 38 & 35 & 34 \\
\hline ALMERÍA & 41 & 38 & 36 & 37 & 35 & 35 & 23 & 32 & 29 & 35 \\
\hline BURGOS & 34 & 37 & 39 & 34 & 37 & 40 & 28 & 33 & 44 & 37 \\
\hline LA RIOJA & 37 & 35 & 38 & 35 & 39 & 41 & 44 & 35 & 43 & 38 \\
\hline CÁDIZ & 52 & 42 & 37 & 43 & 41 & 36 & 37 & 39 & 36 & 39 \\
\hline POLITÉCNICA DE MADRID & 45 & 33 & 31 & 42 & 30 & 30 & 50 & 41 & 40 & 40 \\
\hline CORUÑA. A & 38 & 43 & 44 & 31 & 40 & 42 & 36 & 44 & 42 & 42 \\
\hline JAÉN & 50 & 45 & 41 & 41 & 44 & 38 & 42 & 47 & 37 & 42 \\
\hline VALLADOLID & 40 & 44 & 42 & 32 & 38 & 39 & 56 & 56 & 46 & 42 \\
\hline PALMAS (LAS) & 46 & 47 & 48 & 40 & 43 & 45 & 29 & 37 & 39 & 43 \\
\hline HUELVA & 54 & 51 & 47 & 49 & 45 & 43 & 41 & 36 & 38 & 45 \\
\hline CARLOS III & 42 & 49 & 46 & 46 & 51 & 46 & 35 & 43 & 49 & 46 \\
\hline LEÓN & 49 & 46 & 43 & 50 & 47 & 44 & 45 & 48 & 41 & 46 \\
\hline POLITÉCNICA DE CARTAGENA & 44 & 39 & 40 & 52 & 54 & 47 & 48 & 50 & 47 & 47 \\
\hline U.N.E.D. & 48 & 48 & 45 & 56 & 42 & 48 & 55 & 55 & 56 & 48 \\
\hline CARDENAL HERRERA-CEU & 55 & 55 & 55 & 45 & 50 & 53 & 34 & 42 & 48 & 50 \\
\hline DEUSTO & 43 & 50 & 53 & 47 & 52 & 54 & 38 & 45 & 50 & 50 \\
\hline EUROPEA DE MADRID & 53 & 54 & 54 & 48 & 46 & 50 & 39 & 46 & 51 & 50 \\
\hline PÚBLICA DE NAVARRA & 51 & 52 & 51 & 44 & 48 & 49 & 52 & 52 & 45 & 51 \\
\hline RAMÓN LLULL & 36 & 40 & 49 & 54 & 49 & 51 & 53 & 53 & 54 & 51 \\
\hline MONDRAGÓN & 56 & 56 & 56 & 51 & 53 & 55 & 47 & 49 & 52 & 53 \\
\hline PONTIFICIA DE COMILLAS & 47 & 53 & 52 & 53 & 55 & 56 & 51 & 51 & 53 & 53 \\
\hline SAN PABLO-CEU & 35 & 41 & 50 & 55 & 56 & 52 & 54 & 54 & 55 & 54 \\
\hline
\end{tabular}

Table 6 Ranking of Spanish universities during the period 2006-2010 according to the multidimensional prestige $M W(k)$ of influential fields, for different selections of $k$ and thresholds $z_{j}$.

Recall that if we choose larger values for thresholds $z_{j}$ (e.g., only the top $20 \%$ of the score distribution are dimension-specific influential), we have that the ranking of Spanish universities will be based on more elitist principles. 
By the contrary if the values of thresholds $z_{j}$ decrease (e.g., the top $40 \%$ of the score distribution are dimension-specific influential), it follows a more comprehensive analysis.

Looking for a general pattern of rankings across all the above selections for $k$ and thresholds $z_{j}$, from Table 5 we have that the top ten Spanish universities were (based on the Median rank): (1) Barcelona; (2) Autónoma de Barcelona; (3) Complutense de Madrid; (4) Valencia; (5) Autónoma de Madrid; (6) Pompue Fabra; (7) Granada; (8) Santiago de Compostela; (9) Sevilla; and (10) Zaragoza.

This result is congruent with those from other academic ranking studies (Shanghai Jiao Tong University, 2011). It should be pointed out that we have been able to report these results without assigning weights, since the various scores on different dimensions can be combined into a single score that reflects overall quality of a given university. Our ranking follows rigorous methodological criteria and thus may constitute an effective instrument for quality assessment of universities. The three main characteristics of our data were: (1) Internationally comparable data; (2) quantitative and qualitative indicators; and (3) open to verification.

\section{Conclusions}

Here we have presented a comparison of 56 Spanish universities based on the measurement of multidimensional prestige of influential fields of study during the period 2006-2010.

The multidimensional prestige takes into account that several indicators should be used for a distinct analysis of structural changes at the score distribution of field prestige. We argue that the prestige of influential field of study at a given 
university should not only consider one indicator as a single dimension, but in addition take into account further dimensions.

After having identified the multidimensionally influential fields of study at a given university, their prestige scores can be aggregated to produce a summary measure of multidimensional prestige for this university which satisfies numerous properties (following an axiomatic approach).

What are the limitations of the proposed approach? It is not rare that one would like to impose more axioms that are jointly compatible. It may also happen that the summary measure resulting from the original list of axioms is found to react very bad to some significant institution. One must then formalize the characteristics of the particular institution and state an additional axiom that specifies how the criterion should behave in this situation, and finally determine the greatest subset of axioms from the original list that are compatible with the new axiom. Of course, compatibility may hold for several distinct such subsets.

From the results showed in this paper, the top three Spanish universities (during the period 2006-2010) were: (1) Barcelona; (2) Autónoma de Barcelona; and (3) Complutense de Madrid.

In this paper we argue that this type of analysis, for example, may be relevant to the evaluation of research output using objective metrics in several quantitative and qualitative dimensions, which may guide student choice of a university to pursue a graduate degree or funding agencies to make their decisions regarding the allocation of limited funds.

We are developing a publicly available suite of Web-based tools designed to facilitate analysis of Spanish universities using the proposed approach. It will be freely available at: http://cvg.ugr.es/scientometrics 
Acknowledgments. This research was sponsored by the Spanish Board for Science and Technology (MICINN) under grant TIN2010-15157 cofinanced with European FEDER funds.

\section{References}

Chokri Dridi, Wiktor L. Adamowicz and Alfons Weersink, (2010). Ranking of Research Output of Agricultural Economics Departments in Canada and Selected U.S. Universities. Canadian Journal of Agricultural Economics, Vol. 58, 273282. DOI: $10.1111 /$ j.1744-7976.2010.01188.x

N.C. Liu, and Y. Cheng, (2005). Academic ranking of world universities: Methodologies and problems. Higher Education in Europe, Vol. 30(2), pp. 127-136.

G. Buela-Casal, O. Gutierrez-Martinez, M.P. Bermudez-Sanchez, and O. VadilloMuñoz, (2007). Comparative study of international academic rankings of universities. Scientometrics, Vol. 71(3), pp. 349-365.

I.F. Aguillo, J. Bar-Ilan, M. Levene, and J.L.O. Priego, (2010). Comparing university rankings. Scientometrics, Vol. 85(1), pp. 243-256.

J.-C. Billaut, D. Bouyssou, and P. Vincke, (2010). Should you believe in the Shanghai ranking?: An MCDM view. Scientometrics, Vol. 84(1), pp. 237-263.

Daniel Torres-Salinas, Jose G. Moreno-Torres, Emilio Delgado-López-Cózar, Francisco Herrera (2011). A methodology for Institution-Field ranking based on a bidimensional analysis: the IFQ2A index. Scientometrics, Volume 88, pp 771786. DOI 10.1007/s11192-011-0418-6

Richard Dusansky and Clayton J. Vernon, (1998). Rankings of U.S. Economics Departments, Journal of Economic Perspectives, Vol. 12 (1), pp. 157-170. 
J.A. Garcia, Rosa Rodriguez-Sanchez, and J. Fdez-Valdivia (2011a). Overall prestige of journals with ranking score above a given threshold. Scientometrics, Volume 89, Number 1, 229-243, DOI: 10.1007/s11192-011-0442-6

J.A. Garcia, Rosa Rodriguez-Sanchez, and J. Fdez-Valdivia (2011b). Ranking of the subject areas of Scopus. Journal of the American Society for Information Science and Technology, Volume 62, Issue 10, pp. 2013-2023.

A. Peichl, and N. Pestel, (2010). Multidimensional Measurement of Richness: Theory and an Application to Germany. IZA Discussion Paper, No. 4825.

A. Sen, 1976. Poverty: An Ordinal Approach to Measurement. Econometrica, Vol. 44(2), pp. 219-231.

N. Takayama, 1979. Poverty, Income Inequality, and Their Measures: Professor Sen's Axiomatic Approach Reconsidered. Econometrica, Vol. 47(3), pp. 747-759.

A. Peichl, T. Schaefer, and C. Scheicher, 2008. Measuring Richness and Poverty: A Micro Data Application to Europe and Germany. IZA Discussion Paper, No. 3790.

J.A. Garcia, Rosa Rodriguez-Sanchez, J. Fdez-Valdivia, and J. Martinez-Baena (2011c). On first quartile journals which are not of highest impact. Scientometrics (15 October 2011), pp. 1-19. doi:10.1007/s11192-011-0534-3

S. Alkire, and J. Foster, (2008). Counting and multidimensional poverty measurement, Working Paper No. 7, Oxford Poverty and Human Development Initiative (OPHI).

L. Bornmann, and H.-D. Daniel, (2008). Selecting manuscripts for a high-impact journal through peer review: A citation analysis of communications that were accepted by Angewandte Chemie International Edition, or rejected but published elsewhere. Journal of the American Society for Information Science and 
Technology, Vol. 59(11), pp. 1841-1852.

D. Aksnes, (2003). Characteristics of highly cited papers. Research Evaluation, Vol. 12, pp. 159-170.

D. Aksnes, and G. Sivertsen, (2004). The effect of highly cited papers on national citation indicators. Scientometrics, Vol. 59, pp. 213-224.

J. E. Hirsch, (2005). An index to quantify an individual's scientific research output. Proceedings of the National Academy of Sciences USA, Vol. 102(46), 1656916572.

Shanghai Jiao Tong University, ( 2011). Academic ranking of world universities (ARWU). http://www. arwu.org/index.js. Accessed Dec 2011.

\section{A Appendix: Set of Axioms}

A first axiom states that a field of study at the given university which is not multidimensionally prestigious should not influence a summary measure of the overall prestige of multidimensionally influential fields.

Axiom 1. Given two configurations of dimension-specific scores $\mathbf{X}$ and $\mathbf{X}^{\prime}$ of the same size $n \times d$ where the scores of multidimensionally influential fields at the university are the same in both cases, the summary measure of the multidimensional prestige of influential fields measured on either configuration should give the same value.

Now, a second axiom can be justified on the idea that small changes in the configuration of dimension-specific scores for multidimensionally influential fields of study shall not lead to discontinuously large changes in the summary measure of multidimensional prestige.

Axiom 2. The summary measure of the multidimensional prestige of influential fields at a given university should be a continuous function of dimension-specific scores for multidimensionally influential fields. 
In the following, a third axiom states than an increment in some dimension-specific score (above the corresponding threshold $z_{j}$ ) for a multidimensionally influential field of study shall increase the summary measure.

Axiom 3. An index of multidimensional prestige of influential fields should increase whenever some dimension-specific score (above threshold $z_{j}$ corresponding to that dimension) rises for a multidimensionally influential field of study.

Next an axiom states a property of subgroup decomposability. That is, the index has to be additively decomposable, i.e., the index of overall prestige is a weighted sum over several subgroups of fields of study in which the complete set $U$ can be partitioned.

Axiom 4 The overall prestige of multidimensionally influential fields can be decomposed into the weighted sum of subgroup-prestige indices.

And the following axiom requires that the summary measure of multidimensional prestige of influential fields shall increase after a progressive transfer (from a more influential field of study to a less prestigious one) of domain-specific scores above the corresponding threshold $z_{j}$ between two multidimensionally influential fields at the university.

Axiom 5 An overall prestige index should increase when a rank-preserving progressive transfer (above the corresponding domain-specific threshold) between two multidimensionally influential fields at a given university takes place.

\section{B Appendix: Proof of Theorem 1}

Proof Given a configuration $\mathbf{X}$, let $M W$ be a normalized weighted sum of the dimensionspecific scores in $\mathbf{X}$ using weighting function $f$

$$
M W=\frac{1}{n \times d} \sum_{i=1}^{n} \sum_{j=1}^{d} f\left(\frac{x_{i j}}{z_{j}}\right)
$$

where we have that $f$ should be a continuous function for multidimensionally influential fields of study in order to satisfy Axiom 2, i.e., to verify that small changes in the configuration of dimension-specific scores (for multidimensionally influential fields at the university) shall not lead to discontinuously large changes in the summary measure $M W$. 
But also it follows that weighting function $f$ should be a strictly increasing function for multidimensionally influential fields of study at the university, since Axiom 3 states that an increment in some dimension-specific score (above the corresponding threshold $z_{j}$ ) for a multidimensionally influential field shall increase the summary measure of multidimensional prestige $M W$

From Axiom 1, a field of study which is not multidimensionally prestigious should not influence the overall prestige $M W$, i.e., $M W$ is independent of the dimension-specific scores for fields of study at the given university which are not multidimensionally influential. Hence to fulfill Axiom 1 we have that

$$
f\left(\frac{x_{i j}}{z_{j}}\right)=0
$$

for all $i$ such that $\phi_{i}(\mathbf{z} ; k)=0$; where $\phi_{i}(\mathbf{z} ; k)$ equals to one if field $s_{i}$ is multidimensionally prestigious and zero otherwise, as given in equation (4).

Now, from Axiom 4, the summary measure $M W$ can be decomposed into the weighted sum of subgroup prestige indices. Thus it follows that the measure $M W$ has to be additively decomposable.

Finally, following Axiom 5, the summary measure of multidimensional prestige $M W$ should increase after a progressive transfer (from a more influential field of study to a less prestigious one) of domain-specific scores above the corresponding threshold $z_{j}$ between two multidimensionally influential fields at the university under consideration. Hence we have that weighting function $f$ has to be concave for multidimensionally influential fields, and thus, the relative dimension-specific scores $\frac{x_{i j}}{z_{j}}$ then have to be transformed by a function that is concave on $(1, \infty)$ for multidimensionally influential fields of study.

For example, given a multidimensionally influential field $s_{i}$, we have that

$$
f\left(\frac{x_{i j}}{z_{j}}\right)=\left(1-\left(\frac{z_{j}}{x_{i j}}\right)^{\beta}\right) \cdot \phi_{i}(\mathbf{z} ; k)
$$

is concave for $x_{i j}>z_{j}$ and $\beta>0$.

To sum up, following Axiom 1 through Axiom 5, the summary measure $M W$

$$
M W=\frac{1}{n \times d} \sum_{i=1}^{n} \sum_{j=1}^{d} f\left(\frac{x_{i j}}{z_{j}}\right)
$$


shall satisfy that $f: R_{+} \rightarrow[0,1]$ is a strictly increasing and concave function on $(1, \infty)$ for multidimensionally influential fields $s_{i}$ at the given university.

Following (Peichl and Pestel, 2010), if we define weighting function $f$ as:

$$
f\left(\frac{x_{i j}}{z_{j}}\right)=\left(1-\left(\frac{z_{j}}{x_{i j}}\right)^{\beta}\right)_{+} \cdot \phi_{i}(\mathbf{z} ; k)
$$

where $(v)_{+}=\max (v, 0)$, we obtain a summary measure of the multidimensional prestige of influential fields, that resembles equation (10) satisfying Axiom 1 through Axiom 5, since $f$ being defined as given in equation (16) it is a strictly increasing and concave function $f: R_{+} \rightarrow[0,1]$ on $(1, \infty)$ for multidimensionally influential fields $s_{i}$. 